\title{
HIV seroconversion during pregnancy and the need for pre-exposure prophylaxis (PrEP)
}

This article was published in the following Dove Press journal: HIVIAIDS - Research and Palliative Care

\author{
Carmen D Zorrilla' \\ Fiorella Reyes Báez' \\ Karolyn González Colón ${ }^{2}$ \\ Jessica Ibarra² \\ Iris García ${ }^{2}$ \\ Ana M Mosquera ${ }^{2}$ \\ 'Obstetrics and Gynecology \\ Department, University of Puerto \\ Rico School of Medicine, San Juan, \\ Puerto Rico; ${ }^{2}$ Maternal Infant Studies \\ Center (CEMI), Obstetrics and \\ Gynecology Department, University \\ of Puerto Rico School of Medicine, \\ San Juan, Puerto Rico
}

\begin{abstract}
The reduction in the mother-to-infant transmission of HIV has been among the early successes of care and treatment of women living with HIV. Prenatal HIV counseling and testing, the availability of diverse antiretroviral therapies, elective cesarean section, and the use of formula milk have significantly reduced the mother-to-infant transmission in the USA and Europe. We are presenting two cases of seroconversion during pregnancy, identified during labor and delivery, of women who received risk reduction counseling and serial HIV testing during pregnancy. Because there are no guidelines for (or easy access to) the use of pre-exposure prophylaxis (PrEP) in pregnancy, they were offered other strategies for prevention including risk reduction counseling, condoms, and serial HIV testing. These cases support the use of PrEP during pregnancy. Both infants were negative and the women are currently receiving long-term highly active antiretroviral therapy. One of them recently delivered another infant. After these two women seroconverted, we decided to offer PrEP to all pregnant women presenting for care who report having an HIV positive partner. During the period 2012-2014, we treated ten HIV negative pregnant women who were partners of HIV positive men. Since 2015, we have seen 20 pregnant women in HIV discordant relationships. Of those, seven received PrEP. No seroconversions have been observed among the pregnant women on PrEP. Although small numbers, seroconversion during pregnancy was observed in two of $13(15 \%)$ of the pregnant women in HIV-discordant relationships seen in our clinic, excluding those treated with PrEP. Given the safety data and experience with tenofovir and emtricitabine among pregnant women living with HIV, we believe PrEP should be offered in pregnancy and that guidelines should reflect this option as an additional strategy to reduce risks during pregnancy and to further reduce infant HIV transmission risk.
\end{abstract}

Keywords: PrEP, pregnancy, HIV, HIV seroconversion

\section{Introduction}

The worldwide reduction in the mother-to-infant transmission of HIV has been among the great stories of success for the care and treatment of women living with HIV during pregnancy. Prenatal HIV counseling and testing, and the availability of diverse antiretroviral therapies, elective cesarean section, and the use of infant formula have now reduced the transmission to less than $2 \%$ in the USA and Europe. ${ }^{1}$

This has been possible because of early identification of pregnant women living with HIV and the availability of treatment. Nevertheless, pregnant women might have increased susceptibility to acquiring HIV infection and have been documented to have higher rates of HIV seroconversion. ${ }^{2,3}$ The physiological and immunologic alterations during a normal pregnancy might explain such susceptibility. ${ }^{14}$
Correspondence: Carmen D Zorrilla Obstetrics and Gynecology, University of Puerto Rico School of Medicine, PO Box 365067 San Juan, Puerto Rico 00936-5067

Tel +l 7877535913

$\mathrm{Fax}+|78777| 4739$

Email carmen.zorrilla@upr.edu 
Two relatively recent studies have documented higher rates of seroconversion during pregnancy among women at risk for HIV. An analysis of the seroconversion rates for participants in the Partners in Prevention HSV/HIV Transmission Study showed higher seroconversion risks for pregnant women. The study enrolled 3,408 HIV-1 sero-discordant couples from seven African countries (Botswana, Kenya, Rwanda, South Africa, Tanzania, Uganda, and Zambia). HIV-1 incidence in women was 7.35 versus 3.01 per 100 person-years during pregnant and non-pregnant periods (HR $2.34,95 \%$ CI $1.33-4.09){ }^{2}$ Another study with an analysis of a national sample of women and infant pairs in South Africa, revealed that pregnant women who knew the baby's father was HIV positive had the highest risk for HIV seroconversion in pregnancy. ${ }^{3}$

We now have biomedical HIV prevention strategies that have been recommended to populations at high risk including HIV sero-discordant couples, of which pre-exposure prophylaxis (PrEP) has been demonstrated effective and recommended for diverse groups including men who have sex with men (MSM) and heterosexual women at high risk such as commercial sex workers.

\section{PrEP}

PrEP with daily oral tenofovir disoproxil fumarate (TDF)/ emtricitabine (TDF/FTC) has been shown, in randomized controlled trials, to be effective for prevention of HIV acquisition among diverse populations. ${ }^{4}$ These include MSM, heterosexual men and women, and injection drug users. ${ }^{4}$ In addition, the use of PrEP was not associated with increased pregnancy-related adverse events or hormonal contraception effectiveness changes, in a systematic review and metaanalysis of PrEP. ${ }^{4}$ In 2015 and 2016, the World Health Organization (WHO) included recommendations to offer PrEP to populations at risk with the aim of reducing new infections. The current guideline recommends the use of oral PrEP containing TDF as an added preventive approach for people at risk of HIV infection. They further defined substantial risk as an HIV incidence of three per 100 person-years or higher.

Even though acknowledging that pregnancy might present an increased risk for HIV acquisition and transmission to infants, the WHO guidelines do not specifically recommend such intervention during pregnancy and suggest the need for further research. They suggest that the risks and benefits for continuing PrEP during pregnancy should be evaluated with additional research. ${ }^{5}$ In another study of sero-discordant couples, The Partners PrEP study, women who used PrEP in the early weeks of their pregnancy experienced pregnancy loss and pre-term birth at similar rates, whether they were on PrEP or placebo. The rates of preterm birth, congenital anomalies, and infant growth throughout the first year of life did not differ significantly for infants born to women who received PrEP versus placebo, as reported by the authors. ${ }^{6}$

While PrEP has been recommended to discordant couples for the prevention of HIV transmission, no guidelines or recommendations are currently in place for HIV seronegative pregnant women at risk for HIV. With PrEP, we have an effective intervention and can identify people who need such intervention with rapid testing implemented in multiple settings including clinics that provide reproductive health services to people living with HIV and their partners. Such is the case of HIV sero-discordant HIV couples when the female partner is seronegative and pregnant.

When offered in a clinical setting, women accept the treatment for prevention during pregnancy and post-partum in large proportions. A recent report of clinical experiences with PrEP offered to pregnant women in two USA cities (San Francisco and New York) described acceptance of PrEP by a majority $(67 \%)$ of women it was offered to $(n=24){ }^{7}$

\section{The clinic}

The Maternal Infant Studies Center (CEMI - Spanish acronym) is the largest clinic for women living with HIV, established in 1987 . We have seen more than 2,500 women at the clinic and provide care to 50 new pregnant women living with HIV every year.

Moreover, CEMI is the only clinic that specializes in gynecologic care for HIV infected women in Puerto Rico in addition to obstetrical care. Because of this, we receive constant referrals from allover the island for the treatment of HIV in women, prenatal care, and for the management of the many gynecological conditions common in these patients. We also facilitate a visit for the pregnant women in care to the pediatric clinic, to support the future retention of the infants and children. For the past few years, we have been providing PrEP to both men and women at risk of HIV as per the WHO and United States Public Health Service (USPHS) guidelines. ${ }^{5}$

We are presenting two cases of seroconversion during pregnancy, identified during labor and delivery, of women who received risk reduction counseling and serial HIV testing during pregnancy. One of these women was receiving care from another provider and we met her during the postpartum period. Because there were no guidelines for the use of PrEP in pregnancy, they were offered other strategies for prevention, including risk reduction counseling, condoms, and serial HIV testing. In addition, we are presenting some 
of these data to support the use of PrEP during pregnancy. Both infants received neonatal oral Zidovudine (ZDV) for 6 weeks as per the pediatric recommendations for exposed infants. Both infants are HIV negative and the mothers are currently receiving long-term highly active antiretroviral therapy (HAART). Based on these two cases, we changed our practice of PrEP use during pregnancy.

Both patients provided written informed consent for the case details to be published and UPR Medical Sciences Campus approved this study (IRB number: 1350894).

\section{Case report I}

Case of a 21-year-old female (G3P1021) who has a past medical history of bronchial asthma. This patient received obstetric care from other providers and we learned of her seroconversion during pregnancy upon the evaluation of an abnormal post-partum Pap smear. She was diagnosed on January 9, 2015. The patient's partner was a perinatally HIV infected man. She was seronegative for HIV during pregnancy, followed up with regular testing at every trimester by her provider. A rapid test performed during labor admission was positive, and she was treated with ZDV IV and a cesarean section was performed at a Community Hospital in Caguas, Puerto Rico on January 9, 2015.

She delivered a baby boy who eventually was confirmed as HIV negative. The mother's rapid HIV test was confirmed post-partum. Laboratory results on January 23, 2015 reported HIV-1/HIV-2 antibody differentiation test reactive antibody to HIV-1; non-reactive HIV-2 and HIV-1/HIV-2 antigen/antibody test reactive. The patient was placed on HAART post-partum, receiving atazanavir/cobicistat and FTC/TDF (Evotaz and Truvada). We came to know the patient after her delivery when she was referred due to the abnormal Pap smear. As per our standard management, all exposed infants receive oral ZDV prophylaxis for 6 weeks postnatally and are followed up with serial HIV DNA PCR testing. This infant was negative.

She was followed up by her primary physician and had an abnormal Pap smear on April 30, 2015, reported as low-grade squamous intraepithelial lesion (LSIL) and human papillomavirus (HPV) 18 positive. Six months later, the Pap smear was repeated on November 3, 2015, and was reported as atypical squamous cells of undetermined significance (ASCUS) and HPV high risk positive. Viral load at the time was 89 copies/ $\mathrm{mL}$ and absolute CD4 count was $197 \mathrm{cell} / \mu \mathrm{L}$. The patient was referred to our clinic for further evaluation. A colposcopy and biopsies were performed on December 2, 2015. Cervical biopsy reported LSIL and endocervical curettage showed loose endocervical cells with no significant pathologic changes. She was treated with cryosurgery on December 16, 2015. She was receiving Depo Provera as contraception and the last dose was given in August 2015. In November 2016, she presented with a gravid uterus with approximately 15 weeks gestation by last menstrual period: July 25, 2016. Her absolute CD4 was 397 cells $/ \mu \mathrm{L}$ and viral load was $<40$ copies $/ \mathrm{mL}$. The patient decided not to continue with the pregnancy for personal reasons. She is being followed up by her primary provider.

\section{Case report 2}

Case of a 17-year-old female (G1P0A0) primigravida who was evaluated at our clinic on May 26, 2015. On her first prenatal care visit, she presented with an intrauterine pregnancy of 14 weeks gestation; estimated due date: February 4, 2015 (first trimester ultrasound). Her partner was a perinatally infected HIV seropositive man treated with HAART at another clinic, with no medical complications. We do not have the names of the original drugs prescribed to her partner. He reported good adherence and being undetectable but we had not obtained copies of his laboratory results. She had a past medical history of bronchial asthma and had been seronegative for HIV on prior testing.

During prenatal care, she tested positive for Chlamydia, which was treated; and had a positive triple screen for aneuploidy on June 11, 2015. She was referred for a level II ultrasound that was within normal limits at 23 weeks of gestation. The patient was followed closely over eight prenatal care visits. At every visit, safe sex was recommended and condoms were provided. She was seronegative for HIV during pregnancy, followed up with regular testing at every trimester. A rapid HIV1/2 test performed during admission for early labor was positive on November 6, 2015, at the University Hospital in San Juan, Puerto Rico. Further HIV-1 Western blot and HIV1/2 antibodies were positive, which confirmed seroconversion on November 7, 2015, with a viral load of 514 copies $/ \mathrm{mL}$. The patient was treated with raltegravir/Combivir and given ZDV IV since she was in early labor. However, she had spontaneous rupture of membranes and a cesarean section was performed on November 8, 2017. After genotyping was ordered, she was treated with raltegravir/Combivir and Truvada at the hospital and discharged home with further follow-up at our clinic. Her baby girl was confirmed as HIV negative after further testing.

Three months after delivery, she had a positive pregnancy test on February 3, 2016, and underwent an elective abortion and placement of a progesterone intrauterine device (IUD). She continued to have viremia and on May 18, 2016, her HIV RNA viral load was reported as 4,806 copies/mL, CD4 count of 859 cells $/ \mu \mathrm{L}$, and white blood cells at $4.7 \times 10^{3}$ cells $/ \mu \mathrm{L}$. Based on the genotype results, the patient was switched to 
elvitegravir/cobicistat/FTC/tenofovir alafenamide (Genvoya) once a day. Close monitoring continued until October 12, 2016, when viral load was found to be undetectable, CD4 count was 862.04 cells $/ \mu \mathrm{L}$, and white blood cell count was 6.2 $\times 10^{3}$ cells $/ \mu$ L. On February 8,2017 , the patient had a positive pregnancy test. Her IUD had been removed at another clinic upon her request. She went to her first prenatal care visit on February 21, 2017, at CEMI. She was found with intrauterine pregnancy of 10 2/7 weeks gestation, a viral load of 3,570 copies $/ \mathrm{mL}$, and CD4 count of 734 cells $/ \mu \mathrm{L}$. She delivered at home, 4 days after hurricane Maria hit Puerto Rico (September 20, 2017), because roads were blocked and she could not reach a hospital on time. At the time of delivery, she had an undetectable VL while on highly active antiretroviral therapy (HAART). The baby girl received neonatal oral ZDV, as per the pediatric standard of care and had serial testing for HIV. She is HIV negative.

\section{Discussion}

There is some evidence in the literature that the drugs used for PrEP (tenofovir and FTC) are safe when used in pregnancy among women living with HIV.

A recent publication by Mugwanya et al, of an oral PrEP trial among HIV negative breastfeeding women in Thika, Kenya, and Kampala, Uganda reported extremely low levels of drug acquired via breastfeeding among infants. They reported median (IQR) time-averaged peak concentrations in breast milk of $3.2 \mathrm{ng} / \mathrm{mL}$ (2.3-4.7) for tenofovir and $212.5 \mathrm{ng} /$ $\mathrm{mL}$ (140.0-405.0) for FTC. They further estimated equivalent doses an infant would ingest daily from breastfeeding as 0.47 $\mu \mathrm{g} / \mathrm{kg}$ (IQR 0.35-0.71) for tenofovir and $31.9 \mu \mathrm{g} / \mathrm{kg}$ (IQR $21.0-60.8)$ for FTC. These would translate to $\mathrm{a}<0.01 \%$ and $0.5 \%$ relative dose when compared to the $6 \mathrm{mg} / \mathrm{kg}$ dose that is proposed for therapeutic treatment of infant HIV infection. Tenofovir was not detected in plasma of $94 \%$ of the infants. ${ }^{8}$ In conclusion, these data suggest that PrEP can be safely prescribed during breastfeeding with minimal infant drug exposure.

In an accompanying commentary, Mofenson discusses the evidence accumulated for the need for PrEP (HIV sero-prevalence among pregnant and lactating women in Sub-Saharan Africa), its efficacy, safety during pregnancy among women living with HIV, and limited breast milk exposure among HIV negative women. ${ }^{9}$ She concludes that the evidence would support the use of PrEP in pregnant and lactating women at risk of HIV infection.

A recent systematic review, regarding the safety of TDF and FTC during pregnancy when used by HIV infected women taking combination antiretroviral treatment is reassuring. Data on teratogenicity related to in-utero exposure to TDF and FTC have also been reassuring, with no increase in congenital anomalies compared to the expected background rate for infants enrolled in the Antiretroviral Pregnancy Registry ( $n=3,007$ and $n=2,326$ to date with first trimester exposure to TDF and FTC, respectively) and in prospective studies of women receiving antiretroviral treatment. ${ }^{10}$ The prevalence of birth defects with first trimester tenofovir exposure was $2.23 \%(67 / 3,007) .{ }^{10}$ IMPAACT $\mathrm{P} 1026 \mathrm{~s}$ is a prospective, non-blinded pharmacokinetic study of HIV infected pregnant women that included a cohort receiving $300 \mathrm{mg}$ TDF once daily. In the analysis of 37 pregnant women receiving TDF-based combination therapy at 30-36 weeks gestation and 6-12 weeks postpartum, the proportion of women exceeding the target of $1.99 \mu \mathrm{g} *$ hour/ $\mathrm{mL}$ (the10th percentile in non-pregnant adults) tenofovir was lower in the third trimester (73\%) than post-partum (84\%). ${ }^{11}$ In a retrospective evaluation of $127 \mathrm{HIV}-1$ infected women receiving HAART during pregnancy, in which 15 women received a TDF-based regimen with a median in-utero TDF exposure of 127 days, tenofovir was found to be a welltolerated component of HAART in this small cohort. ${ }^{12}$ In a European study of 34 women receiving TDF plus FTC in the third trimester and postpartum, tenofovir AUC, peak, and trough levels were all approximately $25 \%$ lower in pregnancy compared to postpartum, but these decreased exposures were not associated with virologic failure. Standard dosing during pregnancy was recommended by the authors. ${ }^{13}$

We present two recent cases of HIV seroconversion of pregnant women who received regular HIV testing during pregnancy because they were partners of HIV positive men who reported being on HAART. These women were not offered PrEP because of lack of guidelines at that moment. In addition, PrEP was unavailable because health care insurance would not cover medications without guidelines and the patients could not afford the cost of PrEP. After these cases were identified, we were able to access PrEP through expanded access programs. The identification of seroconversion during pregnancy within an environment with access to PrEP justifies, in our opinion, the use of PrEP for pregnant partners of men living with HIV. The evidence supports its efficacy and safety during pregnancy.

After these two women seroconverted, we decided to offer PrEP to all pregnant women presenting for care who report having a partner living with HIV. At this time, drugs were also available through expanded access programs. Previously, during the period 2012-2014, we treated ten HIV negative pregnant women who were partners of HIV positive men. Since 2015, we have seen 20 pregnant women in HIV 
discordant relationships. Of those, seven have received PrEP. No seroconversions have been observed among pregnant women on PrEP. Although small numbers, seroconversion during pregnancy was observed in two of $13(15 \%)$ pregnant women in HIV-discordant relationships in our clinic, excluding those treated with PrEP. Given these safety data and experience with TDF and FTC among pregnant women living with HIV, we believe PrEP should be offered in pregnancy and guidelines should reflect this option as an additional strategy to reduce risks during pregnancy and to further reduce infant HIV transmission risk.

Some of the limitations of this manuscript include the fact that these are case reports and the remaining numbers of pregnant women in HIV serodiscordant relationships are based on a single clinic's experience and do not represent a population survey. Nevertheless, our clinic cares for the largest number of pregnant and non-pregnant women living with HIV in Puerto Rico and has the infrastructure and expertise to offer PrEP among different populations.

\section{Disclosure}

Dr Zorrilla receives research funding from the National Institutes of Health: NIAID, NICHD and NIMH; from the US Department of Health and Human Services: HRSA HIV/ AIDS Bureau/Ryan White Program and from the following companies: Gilead, BMS and GSK. The other authors report no conflicts of interest in this work.

\section{References}

1. Townsend CL, Byrne L, Cortina-Borja M, et al. Earlier initiation of ART and further decline in mother-to-child HIV transmission rates, 2000-2011. AIDS. 2014;28(7):1049-1057.
2. Mugo NR, Heffron R, Donnell D, et al. Increased risk of HIV-1 transmission in pregnancy: a prospective study among African HIV-1 serodiscordant couples. AIDS. 2011;25(15):1887-1895.

3. Dinh TH, Delaney KP, Goga A, et al. Impact of maternal HIV seroconversion during pregnancy on early mother to child transmission of HIV (MTCT) measured at 4-8 weeks postpartum in South Africa 2011-2012: a national population-based evaluation. PLoS One. 2015;10(5):e0125525.

4. Fonner VA, Dalglish SL, Kennedy CE, et al. Effectiveness and safety of oral HIV pre-exposure prophylaxis for all populations. AIDS. 2016;30(12):1973-1983.

5. World Health Organization. Consolidated guidelines on the use of antiretroviral drugs for treating and preventing HIV infection: recommendations for a public health approach. Geneva: World Health Organization; 2016. Available from: http://apps.who.int/iris/ bitstream/10665/208825/1/9789241549684_eng.pdf?ua=1. Accessed February 26, 2018.

6. Mugo NR, Hong T, Celum C, et al. Pregnancy incidence and outcomes among women receiving preexposure prophylaxis for HIV prevention: a randomized clinical trial. JAMA. 2014;312(4):362-371.

7. Seidman DL, Weber S, Timoney MT, et al. Use of HIV pre-exposure prophylaxis during the preconception, antepartum and postpartum periods at two United States medical centers. Presented at: 6th International Workshop on HIV \& Women; February 20-21, 2016; Boston, MA.

8. Mugwanya KK, Hendrix CW, Mugo NR, et al. Pre-exposure prophylaxis use by breastfeeding HIV-uninfected women: a prospective short-term study of antiretroviral excretion in breast milk and infant absorption. PLoS Med. 2016;13(9):e1002132.

9. Mofenson LM. Tenofovir preexposure prophylaxis for pregnant and breastfeeding women at risk of HIV infection: the time is now. PLoS Med. 2016;13(9):e1002133.

10. apresgistry.com [homepage on the Internet]. The antiretroviral pregnancy registry. North Carolina: INC Research; [cited July 15, 2017]. Available from: www.apregistry.com. Accessed February 26, 2018.

11. Best BM, Burchett S, Li H, et al. Pharmacokinetics of tenofovir during pregnancy and postpartum. HIV Med. 2015;16(8):502-511.

12. Nurutdinova D, Onen NF, Hayes E, Mondy K, Overton ET. Adverse effects of tenofovir use in HIV-infected pregnant women and their infants. Ann Pharmacother. 2008;42(11):1581-1585.

13. Colbers A, Hawkins D, Gingelmaier A, et al. The pharmacokinetics, safety and efficacy of tenofovir and emtricitabine in HIV-1 infected pregnant women. AIDS. 2013;27(5):739-748.

14. Sappenfield E, Jamieson DJ, Kourtis AP. Pregnancy and susceptibility to infectious diseases. Infect Dis Obstet Gynecol. 2013;2013:752852.
HIV/AIDS - Research and Palliative Care

\section{Publish your work in this journal}

HIV/AIDS - Research and Palliative Care is an international, peerreviewed open access journal focusing on advances in research in HIV, its clinical progression and management options including antiviral treatment, palliative care and public healthcare policies to control viral spread. The journal is included in PubMed. The manuscript man-

\section{Dovepress}

agement system is completely online and includes a very quick and fair peer-review system, which is all easy to use. Visit http://www.dovepress com/testimonials.php to read real quotes from published authors. 